\title{
On the Kleinecke-Shirokov Theorem for families of derivations
}

\author{
by \\ Victor S. Shulman (Vologda) and YurIĬ V. TurovskiI (Baku)
}

\begin{abstract}
It is proved that Riesz elements in the intersection of the kernel and the closure of the image of a family of derivations on a Banach algebra are quasinilpotent. Some related results are obtained.
\end{abstract}

The celebrated Kleinecke-Shirokov Theorem ([7], [14]) states that the elements in the intersection of the kernel and the image of a bounded derivation of a Banach algebra are quasinilpotent. M. Thomas [15] proved that this theorem remains true for unbounded derivations. In general, "image" here cannot be replaced by "closure of the image" and "derivation" by "family of derivations". Indeed, there is an inner derivation of the algebra $B(H)$ of all operators on a Hilbert space $H$ such that the identity operator 1 belongs to the closure of its range [2]; furthermore, if $T$ is an operator which is not the sum of a nonzero scalar multiple of the identity and a compact operator, then $T$ and $1-T$ belong to the images of some inner derivations of $B(H)$ (cf. [4]), whence 1 belongs to the sum of their images (and also to the intersection of their kernels).

Our aim is to show that for Riesz elements (in particular, for Riesz operators) the situation is much better.

We assume that all algebras and spaces in question are complex. Let $X, X_{\alpha}$ be linear spaces for all $\alpha \in \Lambda$ ( $\Lambda$ is some set). For a family $M=\left(T_{\alpha}\right)$ of linear maps $T_{\alpha}: X \rightarrow X_{\alpha}$ we denote by $\operatorname{Ker} M$ the intersection of kernels of all $T_{\alpha}$. If $Y$ is a subspace of $X$ then $M \mid Y$ denotes the family $\left(T_{\alpha} \mid Y\right)$ of restrictions of all $T_{\alpha}$ to $Y$. Also, for a family $N=\left(S_{\alpha}\right)$ of linear maps $S_{\alpha}: X_{\alpha} \rightarrow X$ we denote by $\operatorname{Im} N$ the linear span of the union of their images.

Recall that a derivation of an algebra $A$ is a linear operator $d: A \rightarrow A$ satisfying the Leibniz rule $d(a b)=a d(b)+d(a) b$ for all $a, b \in A$. Given an

2000 Mathematics Subject Classification: Primary 47B47.

Key words and phrases: algebra, derivation, Kleinecke-Shirokov Theorem, Riesz element. 
element $a \in A$, let $L_{a}$ and $R_{a}$ be the corresponding multiplication operators on $A$ defined by $L_{a} x=a x$ and $R_{a} x=x a$ for all $x \in A$. A derivation $d$ of $A$ is called inner if there exists an element $a \in A$ such that $d=L_{a}-R_{a}$; in this case $d$ is usually denoted by ad $a$. An element $a \in A$ is called a finite rank element if $L_{a} R_{a}$ is a finite rank operator on the space $A$. If an algebra $A$ is not unital then the spectrum $\sigma(a)$ of an element $a$ of $A$ is taken with respect to the algebra $A^{\dagger}$ obtained from $A$ by adjoining the identity element 1 . Any derivation $d$ of $A$ uniquely extends to a derivation of $A^{\dagger}$ (one should only set $d(1)=0)$. So in what follows we may assume in our proofs that an algebra in question has the identity element.

We call a Hausdorff linear topology $\tau$ on $A$ admissible if the multiplication on $A$ is separately continuous with respect to $\tau$ (that means that $L_{a}$ and $R_{a}$ are continuous for all $a \in A$ ).

Now let $A$ be a Banach algebra. An element $a \in A$ is called a Riesz element (resp. compact element) if $L_{a} R_{a}$ is a Riesz operator (resp. compact operator) on the Banach space $A$. Another definition of Riesz elements may be found in [3].

We need some simple lemmas. In what follows $B(X)$ is the algebra of all bounded operators on a Banach space $X$.

LEMMA 1. Let $\lambda$ be an isolated point of the spectrum $\sigma(T)$ of an operator $T \in B(X)$ and $Q$ the Riesz projection of $T$ corresponding to $\lambda$. If there exists a projection $P$ in $B(X)$ such that $P T=T P$ and $(T-\lambda) P$ is quasinilpotent then $P=P Q=Q P$.

Proof. As is well known, $Q$ is the limit of a sequence of polynomials in $T$. Hence $P Q=Q P$. Since

$$
Q X=\left\{x \in X: \lim \left\|(T-\lambda)^{n} x\right\|^{1 / n}=0\right\}
$$

(see Section 149 of [11]) and

$$
\left\|(T-\lambda)^{n} P x\right\|^{1 / n} \leq\left\|(T P-\lambda P)^{n}\right\|^{1 / n}\|x\|^{1 / n} \rightarrow 0
$$

as $n \rightarrow \infty$, we conclude that $P x=Q P x$ for every $x \in X$, i.e. $P=Q P$.

The proof of the following lemma is a modification of the proof given in [1] for the case of compact elements.

Lemma 2. Let $A$ be a Banach algebra, and let $a \in A$ be a Riesz element. Then all nonzero points of $\sigma(a)$ are isolated in $\sigma(a)$.

Proof. If we adjoin the identity element to $A$ then $a$ remains a Riesz element. So one may suppose that $A$ is unital. Let $B$ be a maximal commutative subset of $A$ containing $a$. Then, by Theorem 1.6.14 of [10], $B$ is a closed commutative subalgebra of $A$ such that, in particular, $\sigma_{B}\left(a^{2}\right)=\sigma\left(a^{2}\right)$ ( $\sigma_{B}$ is the spectrum with respect to $B$ ). Since $B$ is an invariant subspace for $L_{a} R_{a}$ and $\sigma\left(L_{a} R_{a}\right)$ is countable, $\sigma\left(\left(L_{a} R_{a}\right) \mid B\right) \subset \sigma\left(L_{a} R_{a}\right)$ by Theorem 0.8 
of [9] and therefore all nonzero points of $\sigma\left(\left(L_{a} R_{a}\right) \mid B\right)$ are isolated. On the other hand, $\sigma\left(\left(L_{a} R_{a}\right) \mid B\right)=\sigma\left(L_{a^{2}} \mid B\right)=\sigma_{B}\left(a^{2}\right)=\sigma\left(a^{2}\right)$. Using the spectral mapping theorem (for instance, see Theorem 0.5 of [9]) we find that all nonzero points of $\sigma(a)$ are also isolated.

By virtue of Lemma 2 one may take a Riesz idempotent of a Riesz element of a Banach algebra $A$ corresponding to a nonzero point of the spectrum of the element. It is clear that such idempotents belong to $A$ even if $A$ is not unital.

Lemma 3. Let $A$ be a Banach algebra, and let $a \in A$ be a Riesz element, $\lambda$ a nonzero point of $\sigma(a), p$ a Riesz idempotent of a corresponding to $\lambda$. Then $p$ is a finite rank element.

Proof. It is clear that $(a-\lambda) p$ is quasinilpotent. Hence $L_{(a-\lambda) p}$ and $R_{(a-\lambda) p}$ are also quasinilpotent. Furthermore, $L_{p} R_{p}$ is clearly a projection commuting with $L_{a} R_{a}$, and $\left(L_{a} R_{a}-\lambda^{2}\right) L_{p} R_{p}$ is quasinilpotent because it is the sum of mutually commuting quasinilpotents $L_{(a-\lambda) p} R_{a p}$ and $L_{\lambda p} R_{(a-\lambda) p}$.

We claim that $\lambda^{2} \in \sigma\left(L_{a} R_{a}\right)$. Indeed, suppose to the contrary that $S$ is the inverse operator of $L_{a} R_{a}-\lambda^{2}$. It is easy to see that

$$
p=S^{n}\left[\left(L_{a} R_{a}-\lambda^{2}\right) L_{p} R_{p}\right]^{n} p^{n}
$$

for every integer $n>0$. Since $\|p\| \geq 1$, we obtain

$$
1 \leq\|p\|^{1 / n} \leq\|S\| \cdot\left\|\left[\left(L_{a} R_{a}-\lambda^{2}\right) L_{p} R_{p}\right]^{n}\right\|^{1 / n}\|p\| \rightarrow 0
$$

as $n \rightarrow \infty$, a contradiction.

So $\lambda^{2} \in \sigma\left(L_{a} R_{a}\right)$. Let $Q$ be the Riesz projection of $L_{a} R_{a}$ corresponding to $\lambda^{2}$. Since $L_{a} R_{a}$ is a Riesz operator, $Q$ is an operator of finite rank. By Lemma $1, L_{p} R_{p}=L_{p} R_{p} Q$. Therefore $L_{p} R_{p}$ is an operator of finite rank.

Lemma 4. Let $A$ be a Banach algebra, and let $a \in A$ be a Riesz element, $\lambda$ a nonzero point of $\sigma(a), p$ a Riesz idempotent of a corresponding to $\lambda$. If $d$ is a (not necessarily bounded) derivation of $A$ and $d(a)=0$ then $d(p)=0$.

Proof. Set $T=L_{a}, Q=L_{p}, X_{1}=Q A$ and $X_{2}=(1-Q) A$. Since the map $b \mapsto L_{b}$ is a homomorphism from $A$ into $B(A)$, it follows from the integral representation of Riesz projections and idempotents (see Section 148 of [11]) that $Q$ is a Riesz projection of $T$ corresponding to $\lambda \in \sigma(T)$. Note that $X_{1}=\left\{x \in A: \lim \left\|(T-\lambda)^{n} x\right\|^{1 / n}=0\right\}$ and $X_{1} \cap X_{2}=(0)$.

Since $(a-\lambda) p(=p(a-\lambda) p)$ is quasinilpotent and belongs to the finitedimensional subalgebra $p A p,(a-\lambda) p$ is nilpotent. So there exists an integer $n>0$ such that $(a-\lambda)^{n} p=((a-\lambda) p)^{n}=0$. Hence $(a-\lambda)^{n} d(p)=$ $d\left((a-\lambda)^{n} p\right)=0$ and therefore

$$
(T-\lambda)^{n}((1-p) d(p))=(a-\lambda)^{n}(1-p) d(p)=(1-p)(a-\lambda)^{n} d(p)=0 .
$$


Then $(1-p) d(p) \in X_{1}$, and also $(1-p) d(p)=(1-Q)(d(p)) \in X_{2}$. Therefore $(1-p) d(p)=0$. Since $a d(p)=d(a p)=d(p a)=d(p) a$, we see that $d(p)$ commutes with $p$. Thus $d(p)=p d(p)=d(p) p$. Taking into account

$$
d(p)=d\left(p^{2}\right)=p d(p)+d(p) p,
$$

we deduce that $d(p)=0$.

Lemma 5. Let $A$ be a Hausdorff topological algebra with respect to some topology $\tau$ (i.e. $\tau$ is an admissible topology on the algebra $A$ ). Let $B$ be a subspace of $A$ and $B_{\tau}$ its $\tau$-closure. If $p$ is a finite rank idempotent of $A$ then $p B p=p B_{\tau} p$.

Proof. If $C$ is the $\tau$-closure of $p B p$ then $p B p \subset p B_{\tau} p \subset C$. But $p B p$ is a finite-dimensional subspace of $A$. So $p B p$ is closed by Theorem 1.3.2 of [13] and therefore coincides with $C$.

Theorem 1. Let $D=\left(d_{\alpha}\right)$ be a family of (not necessarily bounded) derivations of a Banach algebra A. Then, for any admissible topology $\tau$, all Riesz elements of $A$ that belong to the intersection of $\operatorname{Ker} D$ with the $\tau$-closure of $\operatorname{Im} D$ are quasinilpotent.

Proof. Let $E_{\tau}$ be the intersection of $\operatorname{Ker} D$ with the $\tau$-closure of $\operatorname{Im} D$. It is clear that $\operatorname{Ker} D$ is a subalgebra of $A$ and that, for any $a \in \operatorname{Ker} D$ and $b \in \operatorname{Im} D$, the elements $a b$ and $b a$ are in $\operatorname{Im} D$. Now if a net $\left(b_{\gamma}\right)$ of elements of $\operatorname{Im} D$ converges to $c$ in the topology $\tau$ then the nets $\left(a b_{\gamma}\right)$ and $\left(b_{\gamma} a\right)$ also consist of elements of $\operatorname{Im} D$ and converge to $a c$ and $c a$ in the topology $\tau$, respectively. If, in addition, $c \in \operatorname{Ker} D$ then $a c, c a \in \operatorname{Ker} D$. This means that $E_{\tau}$ is an ideal of $\operatorname{Ker} D$ and therefore it is a subalgebra of $A$.

Let $E$ be the norm closure of $E_{\tau}$. Then $E$ is a closed subalgebra of $A$. Now if $a \in E$ is a Riesz element and $\sigma(a) \neq\{0\}$ then the Riesz projection $p$ of $a$ corresponding to a nonzero point of the spectrum of $a$ belongs to the closed subalgebra generated by $a$. Hence $p \in E$. Denote by $B$ the subalgebra $p A p$. By Lemma 3, $p$ is a finite rank element of $A$, so $\operatorname{dim} B<\infty$.

Let $W_{\tau}$ be the $\tau$-closure of $\operatorname{Im} D$, and let $W$ be the norm closure of $W_{\tau}$. Since $E \subset W, p$ belongs to $W$ and therefore $p$ is in $p W p$. On the other hand, it follows from Lemma 5 that $p(\operatorname{Im} D) p=p W_{\tau} p=p W p$. Thus $p$ belongs to $p(\operatorname{Im} D) p$, i.e. $p=\sum_{i=1}^{n} p d_{i}\left(a_{i}\right) p$ for some finite subfamily $\left(d_{i}\right) \subset D$. It is easy to see that all operators $d_{i}^{*}$ defined on $B$ by $d_{i}^{*}(c)=p d_{i}(c) p$ for all $c \in B$ are derivations of $B$. It follows from Lemma 4 that $d_{i}(p)=0$ for all $i$. Hence $p=\sum_{i=1}^{n} d_{i}^{*}\left(b_{i}\right)$, where $b_{i}=p a_{i} p \in B$. Then

$$
\operatorname{tr}\left(L_{p} \mid B\right)=\sum_{i=1}^{n} \operatorname{tr}\left(L_{d_{i}^{*}\left(b_{i}\right)} \mid B\right)=\sum_{i=1}^{n} \operatorname{tr}\left(\left[d_{i}^{*}, L_{b_{i}} \mid B\right]\right)=0
$$


because the trace of commutators of finite rank operators is equal to zero. But this is impossible because $L_{p} \mid B$ is the identity operator on $B$ and its trace is equal to $\operatorname{dim} B \neq 0$. So we conclude that $a$ is quasinilpotent.

For the case of inner derivations (with respect to the more special admissible topology $\tau$ ) the result was obtained in [16]. Previously, in [12], for the applications in the theory of representations of functional relations, it was obtained for trace class operators and inner derivations of $B(H)$. An algebraic version of Theorem 1 for matrix algebras may be found in Lemma 2.4 of [5]. For compact operators, one-element families of inner derivations of $B(H)$ and the weak sequential topology, the result was obtained in [6]; the same with respect to the weak topology was obtained in [17]. Some related topics are discussed in [8].

Theorem 2. Let $D=\left(d_{\alpha}\right)$ be a family of (not necessarily bounded) derivations of a Banach algebra $A, B$ a subspace of $A$ and $\operatorname{comm} B$ the commutant of $B$ in $A$. Then, for any admissible topology $\tau$, all Riesz elements of $A$ that belong to the intersection of $\operatorname{comm} B$ with the $\tau$-closure of $\operatorname{Im}(D \mid B)$ are quasinilpotent.

Proof. Let $W_{\tau}$ be the $\tau$-closure of $\operatorname{Im}(D \mid B), b$ a Riesz element of $A$ and $b \in W_{\tau} \cap \operatorname{comm} B$. Suppose to the contrary that there exists a nonzero Riesz idempotent $p$ of $b$ corresponding to a nonzero $\lambda \in \sigma(b)$. Note that $p W_{\tau} p=$ $p \operatorname{Im}(D \mid B) p$ by Lemma 5 , so that $p b p=\sum_{i=1}^{n} p d_{i}\left(a_{i}\right) p$ for some subfamily $\left(d_{i}\right)$ of $D$ and all $a_{i} \in B$. Since $\left[b, a_{i}\right]=0$, we have $\left[a_{i}, p\right]=[b, p]=0$ for each $i=1, \ldots, n$.

Define as above the derivations $d_{i}^{*}$ of $p A p$ by $d_{i}^{*}(a)=p d_{i}(a) p$ for all $a \in p A p$ and $i=1, \ldots, n$. Let $c_{i}=p a_{i} p \in p A p$ for all $i$. Since $p d_{i}(p) p=0$ and $a_{i} p=p a_{i}$, it is easy to check that $d_{i}^{*}\left(c_{i}\right)=p d_{i}\left(a_{i}\right) p$ and therefore $p b p=\sum_{i=1}^{n} d_{i}^{*}\left(c_{i}\right)$. Moreover, it is clear that $\left[p b p, c_{i}\right]=0$ for all $i$. Since $p A p$ is finite-dimensional, all $d_{i}^{*}$ are bounded derivations of $p A p$. Now it is easy to check that

$$
L_{p b p} \mid p A p=\sum_{i=1}^{n}\left[L_{c_{i}} \mid p A p,-d_{i}^{*}\right] \quad \text { and } \quad\left[L_{c_{j}}\left|p A p, L_{p b p}\right| p A p\right]=0
$$

for all $j=1, \ldots, n$. Then, by Theorem $1, L_{p b p} \mid p A p$ is a quasinilpotent operator, hence $p b p$ is a quasinilpotent element of $p A p$ and therefore is nilpotent. Thus there exists an integer $n>0$ such that $b^{n} p=(p b p)^{n}=0$. On the other hand, $L_{p}$ is a Riesz projection of $L_{b}$ corresponding to $\lambda \in \sigma\left(L_{b}\right)$ so that $\sigma\left(L_{b} \mid p A\right)=\{\lambda\}$ and $L_{b} \mid p A$ is invertible. Therefore we obtain a contradiction. So we have $p=0$. This proves that $b$ is quasinilpotent.

The authors are indebted to Professor J. Zemánek for useful comments. 


\section{References}

[1] J. C. Alexander, Compact Banach algebras, Proc. London Math. Soc. (3) 18 (1968), $1-18$.

[2] J. Anderson, Derivation ranges and the identity, Bull. Amer. Math. Soc. 79 (1973), 705-708.

[3] B. A. Barnes, G. J. Murphy, M. R. F. Smyth and T. T. West, Riesz and Fredholm Theory in Banach Algebras, Pitnam Books, London, 1982.

[4] A. Brown and C. Pearcy, Structure of commutators of operators, Ann. of Math. 82 (1965), 112-127.

[5] N. Jacobson, Lie Algebras, Wiley, New York, 1961.

[6] H. W. Kim, On compact operators in the weak closure of the range of a derivation, Proc. Amer. Math. Soc. 40 (1973), 482-486.

[7] D. C. Kleinecke, On operator commutators, ibid. 8 (1957), 535-536.

[8] S. Mecheri, Commutants and derivation ranges, Czechoslovak Math. J. 49 (1999), 843-847.

[9] H. Radjavi and P. Rosenthal, Invariant Subspaces, Springer, Berlin, 1973.

[10] C. E. Rickart, General Theory of Banach Algebras, Van Nostrand, Princeton, 1960.

[11] F. Riesz et B. Sz.-Nagy, Leçons d'analyse fonctionnelle, Akadémiai Kiadó, Budapest, 1972.

[12] Yu. S. Samollenko and V. S. Shulman, On representations of relations of the form $i[A, B]=f(A)+g(B)$, Ukrain. Mat. Zh. 43 (1991), no. 1, 110-114 (in Russian).

[13] H. H. Schaefer, Topological Vector Spaces, MacMillan, New York, 1966.

[14] F. V. Shirokov, Proof of a conjecture of Kaplansky, Uspekhi Mat. Nauk 11 (1956), no. 4, 167-168 (in Russian).

[15] M. P. Thomas, Primitive ideals and derivations on non-commutative Banach algebras, Pacific J. Math. 159 (1993), 139-152.

[16] Yu. V. Turovskiu, On a theorem of Yu. S. Samollenko and V. S. Shulman, in: Proc. I Republic Conf. on Mech. Math. (Baku, 1995), Part II Math., Baku, 1995, 204-208 (in Russian).

[17] R. E. Weber, Derivations and the trace-class operators, Proc. Amer. Math. Soc. 79 (1979), 79-82.

Departament of Mathematics

Vologda State Technical University

15 Lenin Str.

160008 Vologda, Russia

E-mail: shulman_v@yahoo.com
Institute of Mathematics and Mechanics 9 F. Agaev Str. 370141 Baku, Azerbaijan E-mail: info@sinam.net

Received February 20, 2001

Revised version August 10, 2001 